\title{
EFEITOS DO JEJUM INTERMITENTE NA COMPOSIÇÃO CORPORAL E NO RENDIMENTO FÍSICO
}

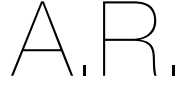
ARTIGO DE REVISÃO

${ }^{1}$ Neuromuscular Research Lab, Faculdade de Motricidade Humana, Universidade de Lisboa, Estrada da Costa, Cruz

1499-002, Cruz Quebrada Dafundo, Lisboa, Portugal

2 CIPER, Faculdade de Motricidade Humana, Universidade de Lisboa, Estrada da Costa, Cruz Quebrada,

1499-002, Cruz Quebrada, Dafundo, Lisboa, Portugal

*Endereço para correspondência:

Joana M Correia

Neuromuscular Research Lab, Faculdade de Motricidade Humana, Universidade de Lisboa,

Estrada da Costa, Cruz Quebrada,

1499-002, Cruz Quebrada, Dafundo, Lisboa, Portugal oanacorreia-19@live.com.p

Histórico do artigo:

Recebido a 14 de junho de 202 Aceite a 15 de dezembro de 2021

\section{EFFECTS OF INTERMITTENTFASTING ON BODY COMPOSITION AND PHYSICAL PERFORMANCE}

Joana M Correia ${ }^{1,2^{\star}}$ iD ; Pedro Pezarat-Correia ${ }^{1,2}$ iD ; Gonçalo V Mendonça $a^{1,2}$ iD

\section{RESUMO}

A comunidade científica manifesta atualmente um elevado nível de interesse no jejum intermitente - períodos de abstinência voluntária da ingestão de energia, variando de várias horas a dias. O jejum intermitente é clinicamente relevante e pode representar uma estratégia não-farmacológica eficaz para melhorar o desempenho físico e a composição corporal. Tem sido estudado principalmente em atletas durante o período religioso do Ramadão e em pessoas predispostas a diminuir a adiposidade corporal sem perda paralela de massa isenta de gordura. O objetivo desta revisão é fornecer uma visão geral do impacto do jejum intermitente durante o Ramadão vs. jejum intermitente não-Ramadão ao nível do rendimento físico e da composição corporal. A literatura evidencia algumas inconsistências ao nível da interação entre o jejum intermitente e o rendimento físico. Contudo, verifica-se que o jejum intermitente não-Ramadão é eficaz para melhorar a potência aeróbia máxima. Não obstante, esta intervenção reduz o desempenho durante sprints repetidos ao longo dos primeiros dias de intervenção. Por outro lado, o jejum intermitente durante o Ramadão diminui a potência aeróbia máxima e isto é mais expressivo durante a segunda metade deste período religioso. Ao contrário, ambas as intervenções são manifestamente inócuas ao nível da força muscular e da capacidade anaeróbia. No que se refere à composição corporal, existe maior consensualidade. De acordo com os dados disponíveis, ambas as intervenções estimulam adaptações benéficas a este nível. Ainda assim, as perdas de massa gorda são mais pronunciadas com o jejum intermitente não-Ramadão.

\section{PALAVRAS-CHAVE}

Desempenho, Massa gorda, Nutrição, Potência aeróbia máxima, Treino

\section{ABSTRACT}

The scientific community currently expresses a high level of interest in intermittent fasting - periods of voluntary abstinence from energy intake, ranging from several hours to days. Intermittent fasting is clinically relevant and may represent an effective nonpharmacological strategy to improve physical performance and body composition. It has been studied mainly in athletes during the religious period of Ramadan and in people predisposed to decrease body fat without loss of fat-free mass parallel. The purpose of this review is to provide an overview of the impact of intermittent fasting during Ramadan vs. non-Ramadan intermittent fasting in terms of physical performance and body composition. The literature shows some inconsistencies in terms of the interaction between intermittent fasting and physical performance. However, non-Ramadan intermittent fasting is found to be effective in improving maximal aerobic power. Nevertheless, this intervention reduces performance during the repeated sprints over the first few days of intervention. On the other hand, intermittent fasting during Ramadan being the maximum aerobic power and this is more expressive during the second half of this religious period. However, both interventions are manifestly innocuous in terms of muscle strength and anaerobic capacity. With regard to body composition, there is greater consensus. According to available data, both interventions encourage beneficial adaptations at this level. Still, fat loss is more pronounced with intermittent non-Ramadan fasting.

\section{KEYWORDS}

Performance, Fat mass, Nutrition, Maximum aerobic power, Training

\section{INTRODUÇÃO}

Tem havido um interesse considerável sobre os efeitos do jejum intermitente (JI) - períodos de abstinência voluntária da ingestão de energia, variando de várias horas a dias ao nível da composição corporal e do rendimento físico (1-6). Existem inúmeras formas de Jl e todas elas envolvem períodos de jejum que se estendem para além das horas de jejum noturno. Implicam ainda uma determinada janela alimentar que se pode ou não acompanhar por restrição calórica $(\mathrm{RC})(7,8)$. A alimentação com restrição de tempo (ART) representa uma forma de Jl. Nesta intervenção, a alimentação é limitada a um número de horas por dia (geralmente $12 \mathrm{~h}$ ou mais) (9). Existem ainda outras formas de Jl que têm emergido na literatura, contudo, estas implicam períodos de jejum que se podem estender a dias completos mais do que uma vez por semana (e.g. dia de jejum alternado, restrição alimentar intermitente) $(9,10)$. O protocolo dietético com dias de jejum alternado 
(DJA) consiste em alternar dias de jejum com dias de alimentação ad libitum - trata-se de um padrão que envolve uma ingestão $\leq 25 \%$ das necessidades energéticas diárias nos dias de jejum ( 500/600 kcal durante 24h), alternando com um ou dois dias de ingestão alimentar ad libitum $(11,12)$. Recentemente, a restrição alimentar intermitente (RAl) tem ganho popularidade na comunidade científica como uma alternativa à restrição alimentar contínua $(\mathrm{RAC})$. A restrição alimentar intermitente pretende estimular melhorias ao nível da composição corporal (13). Inclui períodos de restrição energética alternados com períodos de ingestão ad libitum ou ingestão dietética minimamente restrita, permitindo escolhas alimentares mais amplas (13, 14). Estão descritas várias formas de restrição alimentar intermitente que são atualmente usadas para fins clínicos, incluindo a "dieta 5:2" e a "week-on-week-off" (14-16).

O principal objetivo da generalidade dos regimes de Jl é induzir melhorias na composição corporal - para obter resultados favoráveis ao nível da saúde (e.g. no caso do excesso de peso e da obesidade) e do rendimento físico (17). Como tal, esta tipologia de intervenção tem sido particularmente utilizada em contextos focados na perda de massa gorda (MG) com paralela preservação da massa isenta de gordura (MIG) (9). Há evidência de que o Jl induz reduções no peso corporal e na MG de $\sim 3-7 / 8 \%$ e $\sim 4-15 \%$, respetivamente $(11,18-22)$. Melhorias ao nível do perfil lipidémico, pressão arterial e sensibilidade à insulina têm também sido relatadas de forma consistente $(12,21)$. Mais ainda, de acordo com dados meta-analíticos recentes, o Jl parece ser eficaz para a melhoria ou preservação da MIG quando acompanhado pela prática de exercício físico (23), ou praticado num contexto de ingestão calórica não restritiva (17). Contudo, os estudos centrados na interação entre Jl e rendimento físico têm demonstrado resultados não universais. Schoenfeld et al., verificou que o treino da força praticado em jejum (pós-jejum noturno) pode não ser vantajoso para a perda de gordura e pode mesmo ser prejudicial no que concerne o aumento da massa muscular devido a um potencial aumento da proteólise miocitária (24). No entanto, recentemente, em três ensaios clínicos randomizados que envolveram 8 semanas de treino da força (combinados com uma dieta normal vs. protocolo de Jl), constatou-se que o Jl contribui para a melhoria da composição corporal e do rendimento físico (2, 9, 25). Estes resultados contrastam com o observado na resposta à RAC (e.g. dietas cetogénicas) que tende a induzir algum nível de atrofia muscular esquelética (26). Enquanto Moro et al. não encontraram diferenças entre alimentação com restrição de tempo vs. dieta controlada ao nível do aumento da força máxima dinâmica (uma repetição máxima - 1RM) (2), Tinsley et al. observaram melhorias na força e resistência muscular associadas à alimentação com restrição de tempo, tanto nos membros inferiores como nos membros superiores (9).

O Jl é também utilizado em várias práticas religiosas e tem sido bastante estudado em atletas durante o mês do Ramadão (1, 27). O mês sagrado do Ramadão é o principal período religioso do calendário Islâmico e é uma regra fundamental do Islamismo (28). Os muçulmanos jejuam do nascer ao pôr do sol e, embora possam ingerir alimentos ad libitum após o pôr do sol (refeição lftar) e antes do amanhecer (refeição Sahur), a restrição calórica (com ou sem exclusão de alimentos específicos) é uma característica típica do Jl durante o Ramadão (29). Além disso, a ingestão de líquidos é proibida neste período. Esta abordagem de Jl pode afetar negativamente vários aspetos da saúde humana e do rendimento físico, nomeadamente os relacionados com aspetos psicológicos (e.g. motivação), fisiológicos (e.g. ativação muscular) e bioquímicos (e.g. volume plasmático e reservas de glicogénio) (27, 30, 31). No entanto, de acordo com os dados disponíveis, o jejum de curto prazo (7 dias) não exerce um efeito deletério sobre o desempenho aeróbio $(27,32)$, eficiência da marcha (33) ou potência aeróbia máxima
$\left(\mathrm{VO}_{2 \text { máx }}\right)(28,34-36)$. Por outro lado, períodos mais prolongados de jejum (30 dias) parecem reduzir significativamente o $\mathrm{VO}_{2 \max }$, a resposta inotrópica ao exercício e a cinética de $\mathrm{O}_{2}$ (23, 29, 33, 37-39). A perda de rendimento associada ao JI durante o Ramadão pode relacionar-se com o balanço energético negativo induzido pela restrição calórica, podendo acessoriamente ser reflexo de alterações no ritmo circadiano, privação do sono, stresse psicológico, hipoglicemia e desidratação (que inevitavelmente acompanham este regime específico de JI) (37, 39-42). No entanto, o Jl durante o Ramadão é eficaz para a redução do peso corporal (motivada pelo balanço energético negativo referido anteriormente) e do percentual de MG. Melhora ainda o perfil lipídico individual nos casos em que se regista restrição calórica associada ao mês do Ramadão. A este respeito, os dados disponíveis apontam para diminuições nos níveis séricos de colesterol total e de lipoproteína baixa densidade (LDL), melhorando assim a composição corporal através da maior mobilização de ácidos gordos saturados para fins metabólicos (43-45). No entanto, os efeitos do Jl durante o Ramadão sobre a composição corporal não são inteiramente consensuais. Muito embora alguns estudos apontem para reduções significativas no peso corporal (43, 45-54), muitos outros não demostram quaisquer alterações $(1,29,55-61)$.

Tendo em conta todos estes pontos, esta revisão conceptual tem por objetivo:

1) Resumir as principais conclusões dos estudos centrados sobre $O$ efeito de vários regimes de Jl (ou seja, ART, DJA, RAl e Jl Ramadão) ao nível da composição corporal.

2) Resumir os dados resultantes dos estudos que exploraram o impacto do Jl do Ramadão vs. Jl não-Ramadão (regime ART) sobre o rendimento físico.

\section{METODOLOGIA}

Para o efeito, realizaram-se pesquisas em diversas base de dados (PubMed, Scopus, Sport Discus e Web of Science) tendo combinado termos relacionados com o jejum intermitente (i.e. "intermittent fasting", "alternate-day-fasting", "time-restricted feeding", "time-restricted eating", "periodic fasting", "intermittent calorie restriction", "intermittent energy restriction", "fasted state", e "Ramadan"), rendimento físico (i.e. "muscle strength", "resistance training", "anaerobic capacity", "lactate threshold", "aerobic capacity", " $\mathrm{VO}_{2 \max }$ ", e " $\mathrm{VO}_{2 \text { peak }}$ ") e composição corporal (i.e. "body composition", "body mass", "body mass index" , "Quetelet index", "FFM", "fat-free mass", "fat mass", "fat percentage", "body weight", "waist circumference", "muscle mass", "muscle volume", "bone density"). Além disso, selecionaram-se artigos relevantes das listas de referências, bem como de revisões anteriores. Os critérios de inclusão foram: i) adultos ( $\geq 18$ anos), ii) estudos observacionais ou experimentais, iii) estudos que incluíram alterações nas variáveis de rendimento físico, iv) estudos com qualquer tipo de Jl (isto é, ART, DJA, RAI, RAC ou JI Ramadão) e v) estudos com resultados relacionados com variáveis de composição corporal (i.e., peso corporal, MIG, MG, massa / volume muscular, densidade mineral óssea, circunferência da cintura ou índice de massa corporal - IMC). Excluíram-se todos os estudos com enfoque sobre condições ou patologias específicas (i.e. cancro, patologia tiroideia, diabetes mellitus, cirurgia bariátrica, gravidez, amamentação, nutrição parentérica, doença infetocontagiosa, transplante de órgãos, síndroma de Prader-Willi, síndroma do ovário poliquístico, doença pulmonar obstrutiva crónica ou doença aguda, como infeções ou lesões traumáticas), bem como todos aqueles que implicaram a inclusão de participantes sob o efeito de terapêutica farmacológica. Os artigos de revisão, estudos de caso, protocolos, bem como resumos / artigos de conferências foram também excluídos. 
Identificaram-se 94 artigos ( $k=66$ referentes à composição corporal e $\mathrm{k}=28$ referentes ao rendimento físico) potencialmente relevantes para a presente revisão.

\section{Alterações na Composição Corporal com Jejum Intermitente Não-Ramadão}

As diferentes formas de $\mathrm{Jl}$ parecem exercer um impacto positivo na fisiologia humana, nomeadamente no estado de saúde e na composição corporal (12). Parece que todos as tipologias de Jl induzem alguma perda de peso. Recentemente demonstrou-se que 79\% dos estudos observam uma perda de peso significativa subsequente ao Jl nãoRamadão (17). Como tal, as diferentes formas de Jl (ART, DJA e RAl) são adequadas para a perda ponderal e para a redução de MG corporal, podendo ainda representar uma estratégia eficaz para a manutenção da composição corporal $(18,22,62)$. Segundo os dados disponíveis, sabe-se que estas modificações se relacionam com o efeito do jejum ao nível da mobilização de ácidos gordos, potenciação da lipólise bem como da cetogénese (10).

Contudo, a eficácia de qualquer regime dietético depende intimamente da minimização de perdas da MIG e da maximização da perda de MG. A preservação da MIG é, a este nível, essencial para prevenir recidivas a médio/longo prazo. A mesma depende essencialmente de três fatores: i) balanço energético diário / controlo de calorias ingeridas, ii) qualidade do aporte energético (i.e. ingestão de hidratos de carbono complexos, de proteína completa e de ácidos gordos essenciais) e iii) prática concomitante de exercício físico $(23,63,64)$. Até agora não existe evidência de que determinado protocolo de Jl não-Ramadão seja melhor ou pior do que outro. Acredita-se que a eficácia desta intervenção dependa primordialmente da adaptação individual a determinado tipo de protocolo, bem como do estado ponderal de base (excesso de peso e obesidade) e da variável de composição corporal que se pretende melhorar. Por exemplo, a alimentação com restrição de tempo mostrou ser particularmente eficaz para a indução de aumentos ligeiros na MIG $(25,65)$. Na verdade, tem sido verificado que, quando combinada com o treino da força , a alimentação com restrição de tempo contribui para a preservação da MIG ou minoração de perdas associadas à redução ponderal $(2,9,66)$. Mais ainda, o jejum desencadeia inúmeras respostas endócrinas que podem contribuir para o aumento da taxa metabólica de repouso e preservação da MIG (e.g. aumento dos níveis séricos de noradrenalina e da hormona de crescimento, respetivamente) (67). Para além do que já foi mencionado, a prática de exercício em jejum está associada ao aumento da expressão da sirtuina 1 (SIRT1) e da fosforilação da 5' proteína cinase ativada por monofosfato de adenosina (AMPK), ambas com inúmeros efeitos na expressão de genes envolvidos na biogênese mitocondrial (68). Outro exemplo de protocolo de Jl que também demonstrou contribuir para a melhoria da composição corporal é o protocolo de dias de jejum alternado. Cerca de 38-44\% dos estudos observou diminuições significativas no peso corporal e na MG (17). Verificou-se ainda que a adesão individual a este tipo de protocolo é superior ao observado nos casos de restrição alimentar contínua, tendose ainda constatado uma maior eficácia ao nível da redução ponderal e da resistência periférica à insulina $(62,63)$. Segundo os dados de um destes estudos, o grupo intervencionado pelo protocolo 5:2 diminuiu espontaneamente o aporte calórico de 23 para 32\% nos 2 dias sem restrição (62). É curioso notar que a magnitude da restrição espontânea associada ao Jl atingiu uma expressividade idêntica à registada no grupo intervencionado com recurso à restrição alimentar contínua (restrição coerciva). Como tal, não houve uma hiperfagia compensatória durante os dias/horas de aporte ad libitum - fenómeno que poderia contribuir para uma redução muito significativa da eficácia deste tipo de intervenção.
Segundo as conclusões deste estudo constata-se ainda que, a curto prazo, a eficácia do Jl se sobrepõe à restrição alimentar contínua no que diz respeito à redução ponderal e de gordura corporal (62).

Outro aspeto que se relaciona diretamente com a margem de eficácia deste tipo de intervenção é o estado ponderal de cada individuo no início do tratamento. Foi recentemente demonstrado que, nos casos de excesso de peso e obesidade, o J não-Ramadão contribui para reduções significativas no peso corporal, IMC e MG absoluta. Indivíduos com excesso de peso / obesidade quando realizam um protocolo de JI não-Ramadão reduzem significativamente o peso corporal, o IMC e a MG (kg), quando comparados os resultados pré- pós intervenção vs. controlo. Contudo, os autores não verificaram resultados significativos sobre o impacto do Jl ao nível da MIG após controlar para o efeito estado nutricional enquanto moderador desta análise (17). Segundo a literatura existente, a redução da MIG está frequentemente relacionada com o teor proteico da dieta ingerida. A menor fosforilação do mTOR (mammalian target of rapamycin) no músculo esquelético implica uma diminuição da síntese proteica e um aumento da proteólise muscular $(25,64)$. Estas alterações podem predispor a uma perda acrescida de MIG em indivíduos normoponderais face ao observado em pessoas com excesso de peso ou obesidade. Nestas últimas, uma vez que existe maior acumulação de gordura corporal, o organismo tende a recorrer às reservas adipocitárias para fins energéticos ao invés de recorrer à proteína muscular $(64,69)$. Existem conclusões preliminares que apontam para a ingestão suplementar de aminoácidos durante o J como uma estratégia benéfica para a preservação ou aumento da MIG (25). Em particular, a este respeito, parece que a leucina desempenha um papel fundamental (i.e. estimula a síntese de proteínas através da sinalização do mTOR, modulando assim a transcrição da mensagem genética ao nível dos ribossomas) $(25,69)$.

\section{Alterações na Composição Corporal com Jejum Intermitente Durante o Mês do Ramadão}

Além de ser usado para fins clínicos, o Jl também está também implicado em muitas práticas religiosas. Um exemplo notável é o JlRamadão - a forma de Jl universalmente mais estudada (49). Este tipo de Jl resulta em reduções significativas ao nível do peso corporal, MG, IMC, taxa metabólica de repouso e secreção de hormonas anabólicas (e.g. insulina) $(17,43,45)$. Ao contrário, promove o aumento da secreção de hormonas catabólicas (e.g. adrenalina e glucagon) $(43,70)$. Os efeitos do Jl-Ramadão ao nível da composição corporal são secundários a aumentos na capacidade de mobilização de ácidos gordos saturados para processos metabólicos (44), diminuição inevitável na frequência das refeições (de 3-4/dia para 2/dia) e desidratação coerciva (49, 54, $56,71)$. No entanto, a perda ponderal é mais pronunciada em indivíduos que apresentam valores de IMC mais elevados $(17,46)$.

O Ramadão pode representar uma oportunidade para perder peso corporal, principalmente para nos casos de excesso de peso ou obesidade. É relevante realçar que mais de metade do peso perdido pode resultar de reduções observáveis ao nível da MG (72). A maior eficácia em indivíduos com excesso ponderal parece relacionar-se com uma maior perda de fluidos corporais nestes casos (decorrente de uma maior expressão das reservas de glicogénio) (73). Não obstante, as perdas de MIG podem ainda representar 4,3 a 38,3\% do peso perdido em pessoas com excesso de peso e obesidade (reduções totais de $\sim 20$ a 30\% na MIG) (72). Já em indivíduos normoponderais, o Jl- Ramadão pode implicar perdas de MIG proporcionalmente superiores (acima dos 35\%) (74). Contudo, os resultados meta-analíticos publicados recentemente não corroboram estas conclusões. Revelam, na verdade, que o Jl- Ramadão não impacta a MIG de forma significativa (17). 
Do ponto de vista fisiológico, estes são resultados relevantes uma vez que a MIG desempenha um papel importante na capacidade funcional, na taxa metabólica de repouso e na homeostasia da glicose sanguínea (63).

Conjuntamente, estes resultados sugerem que o Jl-Ramadão é eficaz para a melhoria da composição corporal, principalmente em indivíduos com excesso de peso e obesidade.

\section{Alterações no Rendimento Físico com Jejum Intermitente Não-Ramadão \\ Potência Aeróbia Máxima}

Foi demonstrado que a prática recorrente de exercício de resistência cardiorrespiratória após um período de jejum noturno induz melhorias no $\mathrm{VO}_{2 \max }$ (75-77) e no desempenho de tarefas motoras de alta intensidade com predominância aeróbia (78). Quando combinado com 4 semanas de exercício realizado em cicloergómetro (5 sessões/semana), o JI parece resultar em aumentos suplementares no glicogénio muscular vs. o observado na resposta ao treino físico realizado em regime pósprandial (75). Do ponto de vista fisiológico, estes resultados indicam que o exercício praticado com redução dos níveis séricos de glicose circulante tende a aumentar a capacidade de armazenamento de glicogénio muscular - aspeto bem documentado em pesquisas anteriores (67). A prática de exercício físico em jejum prioriza a utilização dos ácidos gordos livres e dos triglicéridos intramusculares enquanto substratos energéticos, inibindo o metabolismo da glicose $(76,79)$. Adicionalmente, reduz a utilização do glicogénio muscular uma vez que há um aumento da contribuição da oxidação total de gordura durante o exercício submáximo $(67,80)$. No entanto, nem todos os desenhos experimentais que exploraram a interação entre jejum e treino observaram melhorias no $\mathrm{VO}_{2 \text { máx }}(67,76,80)$. No entanto, tendo por base os estudos que obtiveram resultados positivos a este respeito, pode afirmar-se que a prescrição mais eficaz para promover ganhos no $\mathrm{VO}_{2 \text { máx }}$ implica sessões mais frequentes de exercício praticado em jejum (5 ou mais sessões/semana). Ao contrário, para induzir mudanças favoráveis ao nível da eficiência e utilização de substratos energéticos durante exercício físico, poderá ser prescrita uma menor frequência semanal (3 sessões/semana) (67). Independentemente destes pormenores, existe evidência de que o treino em jejum aumenta a capacidade oxidativa do músculo esquelético por intermédio do aumento da atividade de enzimas mitocondriais (e.g. citrato sintase e $\beta$-hidroxiacil coenzima $A$ desidrogenase) (76).

\section{Capacidade Anaeróbia e Força Muscular}

Os efeitos do Jl ao nível do rendimento anaeróbio são diversos. Por exemplo, observou-se uma redução significativa no desempenho durante sprints repetidos durante a fase inicial do JI (primeiros 2 a 3 dias de intervenção) $(81,82)$. Este fenómeno pode relacionar-se com o facto de, a curto prazo, o Jl provocar uma depleção substancial das reservas energéticas ao nível muscular (83). Curiosamente, foi também sugerido que a diminuição do desempenho nos sprints repetidos poderia ser secundária à instalação da fadiga central vs. periférica (84). Pode ainda ser uma consequência mais-ou-menos direta de alterações na perceção subjetiva de esforço durante a realização de exercício em jejum. Hipoteticamente, o aumento da perceção subjetiva de esforço pode levar a uma diminuição da ativação voluntária durante os sprints realizados em regime repetitivo (85).

Segundo a literatura disponível, quando combinado com o treino da força, o Jl é eficaz para atenuar o dano muscular agudo e preservar a massa muscular, contribuindo ainda para a redução da gordura corporal e inflamação $(2,9,25,86)$. Moro et al. mostraram que, embora a alimentação com restrição de tempo reduza as concentrações séricas de androgénios, não se regista qualquer diferença ao nível do ganho de massa muscular após o treino cumprido em regime pré- vs. pós-prandial (2). Em consonância com estes resultados, Tinsley et al. demonstraram ainda que, quando combinada com o treino da força, a alimentação com restrição de tempo (vs. dieta regular) induz ganhos de força muscular nos membros superiores e inferiores, bem como a resistência muscular ao nível dos membros inferiores (9). Mesmo com uma ingestão calórica consideravelmente reduzida ( $650 \mathrm{kcal} / \mathrm{dia}$ ), 8 semanas de alimentação com restrição de tempo não afetaram negativamente a preservação da MIG ou os ganhos de força decorrentes do treino em jovens do sexo masculino (9). Concomitantemente, outros autores demonstraram que a implementação de períodos diários de jejum de 16 horas não compromete os ganhos de força ou potência muscular ao longo de 4 semanas de treino (em indivíduos bem treinados). Os resultados deste estudo apontam mesmo para melhorias na área de secção transversa do músculo vasto lateral e do bicípite braquial após o período de intervenção combinada entre alimentação com restrição de tempo e treino da força (87). Como tal, uma vez que não exerce um impacto negativo sobre a força ou massa muscular, pode concluir-se que a alimentação com restrição de tempo representa uma intervenção viável para atletas envolvidos no treino da força que pretendam alterar a sua composição corporal.

\section{Alterações no Rendimento Físico Decorrentes do Jejum Intermitente Durante o Mês do Ramadão}

Potência Aeróbia Máxima

O Jl realizado durante o mês do Ramadão induz um decréscimo do $\mathrm{VO}_{2 \max }(29,88)$. Uma possível explicação para este fenómeno passa pela desidratação coerciva que acompanha invariavelmente o Ramadão. Esta pode reduzir o volume plasmático, o débito cardíaco máximo e ainda as reservas de intramusculares de glicogénio, afetando assim o $\mathrm{VO}_{2 \max }$ (29). No entanto, é importante notar que apenas se registaram reduções significativas no $\mathrm{VO}_{2 \max }$ durante as fases iniciais do mês do Ramadão. Mais ainda, deve reforçar-se que estes decréscimos foram geralmente pouco expressivos e que se esbateram consideravelmente durante a segunda metade do mês do Ramadão (83). Como tal, parece emergir uma progressiva adaptação metabólica ao jejum ao longo do Ramadão. Por exemplo, o Jl-Ramadão é conhecido por aumentar progressivamente a capacidade individual de oxidação de gordura corporal $(89,90)$. Além de compensar o decréscimo na taxa de oxidação da glicose sérica e da glicogenólise muscular, este efeito fornece um substrato suplementar para a neoglicogénese $(91,92)$. Segundo os estudos realizados neste âmbito, o aumento da capacidade de oxidação da gordura durante o Ramadão não se esgota na condição de repouso, sendo também extensível aos momentos de prática física que envolvam exercício de baixa intensidade $(56,93)$. Assim, numa perspetiva fisiológica, pode afirmar-se que este regime particular de $\mathrm{Jl}$ resulta em algumas adaptações positivas do ponto de vista metabólico. Em conformidade, obteve-se evidência parcial de que a perda ponderal durante o Jl-Ramadão resulta de reduções na MG que, por sua vez, podem são secundárias a um estado aprimorado de utilização lipídica durante o exercício (56). Não obstante, tendo por base uma perspetiva diferente, é também possivel que a perda ponderal secundária a esta tipologia de $\mathrm{Jl}$ se relacione com circunstâncias individuais que variem de acordo com amplos fatores culturais e sociais, bem como com estreitos fatores familiares/domésticos (41). Ainda assim, é importante notar que não foram observadas grandes alterações na composição corporal nos casos em que o Jl exerceu um efeito inócuo sobre o rendimento físico. Em conformidade, é possível que exista uma relação intima entre imutabilidade dos parâmetros de composição corporal 
pós-Jl e estabilidade das medidas de rendimento físico obtidas ao longo do tempo em alguns desses estudos (e.g. na capacidade anaeróbia, conforme observado no estudo de Karli et al. (1)).

\section{Capacidade Anaeróbia e Força Muscular}

Existem dados contraditórios no que se refere à interação entre o rendimento anaeróbio e o Jl durante o Ramadão. Karli et al. demostraram melhorias significativas no teste de Wingate (1). No entanto, Souissi et al. observaram que, embora o desempenho no teste de Wingate (pico e potência média) permanecessem inalterados durante o período da manhã no mês do Ramadão, o mesmo não aconteceu durante período da tarde ou noite (19 e 21h, respetivamente) (94). Nessas circunstâncias, observou-se uma redução de rendimento físico comparativamente ao registado durante o período de controlo. Noutros estudos, explorou-se o impacto desta tipologia de jejum sobre o efeito de treino decorrente de sessões que envolveram a prática repetida de sprints supramáximos $(94,95)$. Aqui, concluiu-se que as melhorias no desempenho pós-treino parecem ser altamente específicas em termos de janela horária. Como tal, apenas se manifestam em períodos diários que coincidam com o regular horário de agendamento do treino (95). Conclui-se assim que a interação entre o Jl-Ramadão e o rendimento anaeróbio é modulado pela hora do dia. Ainda pode concluir-se que pequenas alterações no desempenho anaeróbio decorrentes do JlRamadão podem ser secundárias a alterações na perceção subjetiva de esforço, tempos de reação mais lentos ou alterações na função neuromuscular (causadas pela desidratação, conjuntamente com depleção de glicogénio muscular) $(88,94)$.

O impacto do Jl durante o Ramadão na força muscular parece ser, essencialmente, residual. Embora se tenha mostrado que este regime de JI não afeta a altura do salto vertical (29), outros estudos observaram pequenas reduções na força muscular $(28,31,96)$. Apontam-se diversas razões para este efeito deletério, nomeadamente pequenas reduções na MIG, desidratação ou mesmo alterações no uso de substratos energéticos e na instalação de fadiga durante o exercício. No entanto, Kirkendall et al. relataram que o Jl-Ramadão é eficaz para melhorar a altura do salto vertical durante os períodos da tarde, mas não durante os períodos matinais (83). Estes dados sugerem que a relação entre o Jl-Ramadão e a força muscular podem ser largamente modulados pelos efeitos da ritmicidade circadiana sobre o desempenho desportivo (97). Adicionalmente, existe evidência de que o jejum do Ramadão se acompanha por uma redução geral no aporte de hidratos de carbono; condição compatível com eventual diminuição das reservas de glicogénio muscular (98). A disponibilidade limitada de hidratos de carbono pode prejudicar o bom desempenho físico durante exercícios de alta intensidade (99). Este efeito pode ser ainda mais agravado pela inevitável desidratação que acompanha as horas do jejum no mês do Ramadão $(56,98,100)$. Finalmente, os efeitos do Ramadão ao nível das respostas fisiológicas ao exercício e treino físico são inseparáveis das implicações que esta modalidade de Jl tem sobre as horas de sono e hábitos psicológicos ou sociais (uma combinação de fatores que exercem uma influência negativa sobre o rendimento físico) $(83,101)$.

\section{CONCLUSÕES}

O Jl e o treino físico representam duas intervenções complementares (de índole não-farmacológica) com inúmeros benefícios para a saúde humana. A literatura centrada no impacto desta intervenção sobre a composição corporal revela, genericamente, que as diferentes tipologias de Jl resultam em reduções significativas no peso corporal e na MG (Figura 1). Contudo, a perda de MG é mais pronunciada na resposta ao JI não-Ramadão que, quando combinado com exercício

\section{Figura 1}

Representação esquemática do impacto do jejum intermitente (Jl- Ramadão vs. Jl não- Ramadão) na composição corporal. Mecanismos potenciais que contribuem para a interação entre jejum intermitente e a composição corporal.

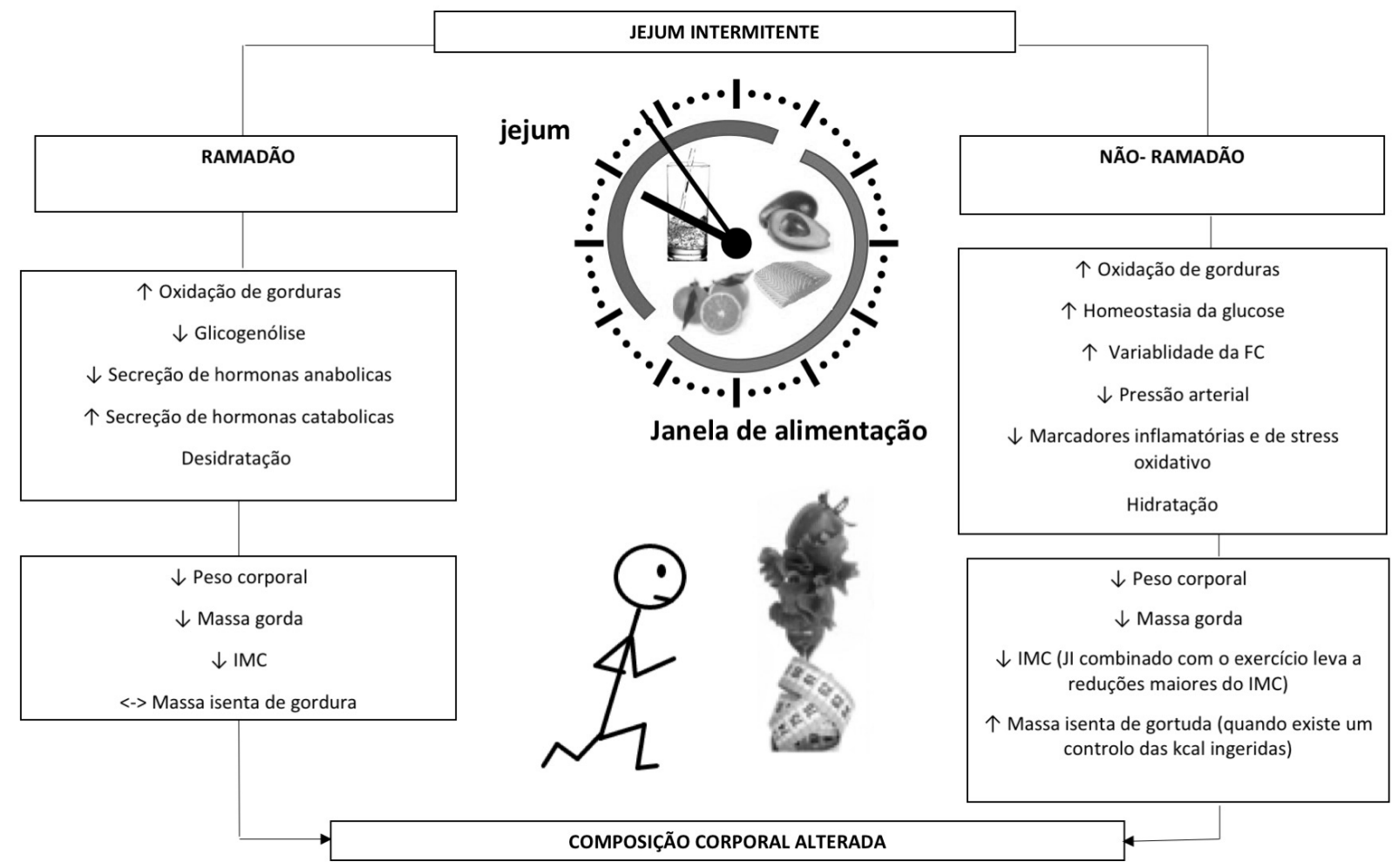


Representação esquemática do impacto do jejum intermitente (Ramadão vs. Alimentação com Restrição de Tempo) no desempenho físico. Mecanismos potenciais que contribuem para a interação mecanicista entre jejum intermitente e desempenho físico.

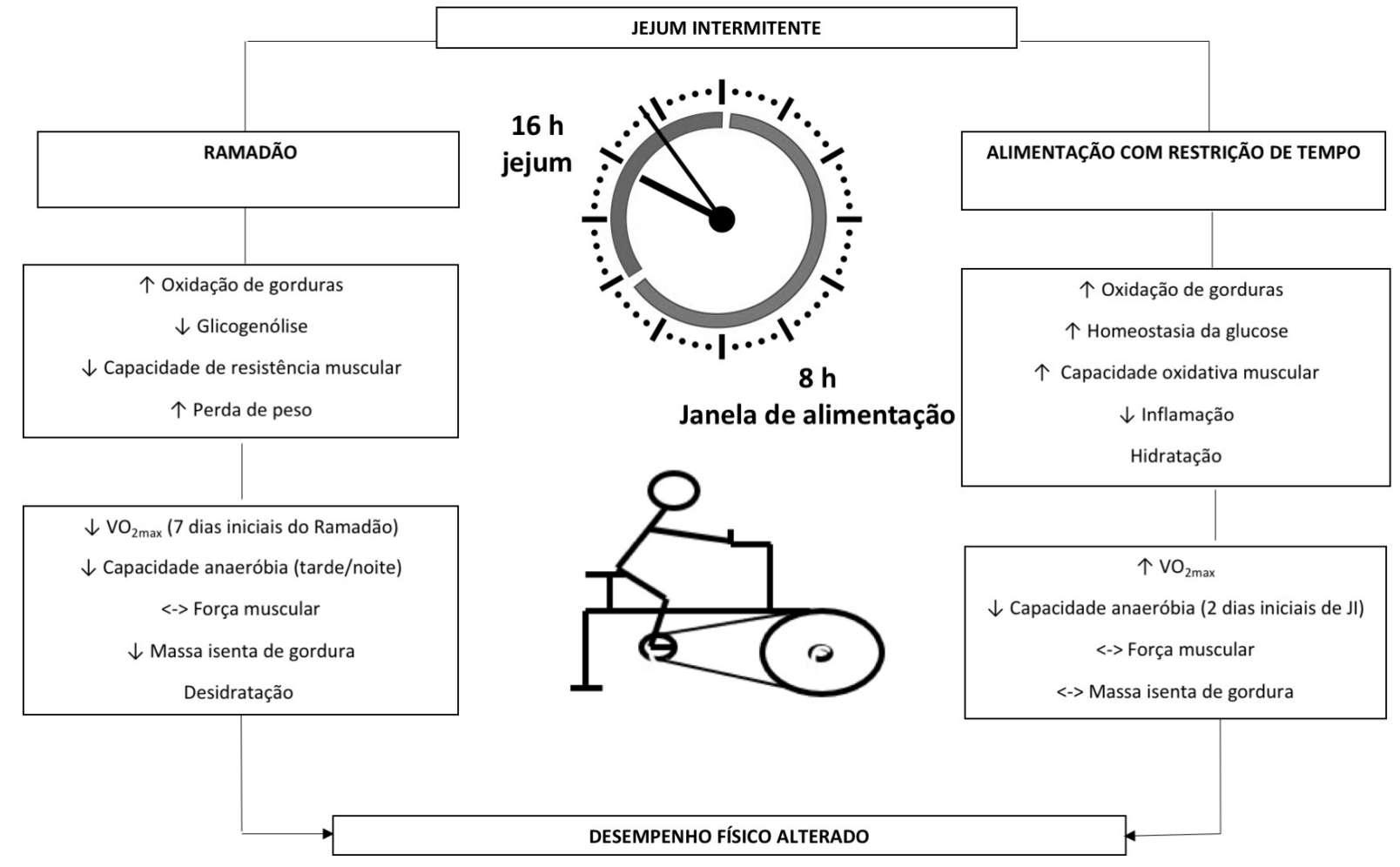

Jl:Jejum Intermitente

físico, leva a reduções ainda mais expressivas no IMC. Constata-se ainda que, aquando da adesão a um regime de Jl não-Ramadão, a salvaguarda de um aporte calórico adequado (aporte não restritivo) contribui de forma decisiva para a indução de pequenos aumentos na MIG. Assim sendo, comparativamente ao verificado no jejum religioso, o Jl não-Ramadão parece ser particularmente eficaz para a indução de benefícios na composição corporal (particularmente, se combinado com exercício físico e ingestão calórica controlada).

No âmbito da interação entre $\mathrm{Jl}$ e rendimento físico, existe maior contrariedade e inconsistência de resultados. Conforme ilustrado na Figura 2, do ponto de vista prático, verifica-se que o Jl não-Ramadão (regime ART) é eficaz para melhorar a potência aeróbia máxima e é viável para atletas envolvidos no treino da força (não afeta negativamente a força nem a massa muscular). Ao contrário, a capacidade anaeróbia tende a diminuir durante o período da tarde durante o mês do Ramadão. Finalmente, o Jl durante o Ramadão acompanha-se de pequenas diminuições no $\mathrm{VO}_{2 \max }$ que tendem a esbater-se durante a segunda metade deste período religioso.

\section{Questões Futuras}

Apesar dos resultados relacionados com os efeitos do Jl (Ramadão vs. não-Ramadão) ao nível da composição corporal serem globalmente favoráveis, ainda assim, é importante que os mesmos sejam interpretados com cautela. Primeiro, as análises foram realizadas com diferentes populações. Segundo, o grau de restrição calórica entre os estudos e os grupos de controlo variou de "dieta normal" a restrição alimentar contínua. Ainda relacionado com este ponto, o perfil nutricional referente às horas de janela alimentar não foi monitorizado. Terceiro, as avaliações de composição corporal foram realizadas com diferentes instrumentos e técnicas de avaliação. Deste modo, é pertinente que a comunidade científica continue a debruçar-se sobre este tema que tem vindo a ganhar popularidade nos últimos anos.

Existe evidência preliminar de que o Jl durante o Ramadão tem uma influência negativa sobre o rendimento físico comparativamente ao sucedido na resposta ao JI não-Ramadão. No entanto, o número de estudos por parâmetro físico analisado é muito limitado e a maioria dos estudos não incluiu grupo de controlo. São também necessários mais estudos que se centrem sobre eventuais interações entre estas abordagens de Jl e o rendimento físico feminino. É ainda fundamental, garantir que o volume e a intensidade do treino sejam bem controlados durante todas as recolhas experimentais. A ingestão de nutrientes deve também ser monitorizada ao longo do tempo, sendo acessoriamente importante determinar o papel moderador das alterações do sono e dos estados de hidratação (de preferência com medidas objetivas) a este respeito. São essenciais mais desenhos experimentais centrados nos efeitos do Jl sobre força muscular - recorrendo a modelos que salvaguardem a ingestão de proteína com qualidade e quantidade suficiente para a promoção da hipertrofia muscular. Também são necessários mais estudos relacionados com os efeitos do $\mathrm{Jl}$ no rendimento físico para descrever os mecanismos e vias metabólicas que asseguram preservação ou mesmo potenciação da prestação atlética durante o Jl. Os fatores fisiológicos responsáveis pela redução da prestação física em algumas circunstâncias também devem ser examinados com maior detalhe.

\section{AGRADECIMENTOS}

Este trabalho foi financiado pela Universidade de Lisboa (bolsa atribuída à JMC - n C00246e). Este trabalho foi parcialmente apoiado pela Fundação para a Ciência e Tecnologia, sob a concessão UIDB / 00447/2020 ao CIPER - Centro Interdisciplinar de Estudo da Performance Humana (unidade 447). 


\section{REFERÊNCIAS BIBLIOGRÁFICAS}

1. Karli U, Guvenc A, Aslan A, Hazir T, Acikada C. Influence of Ramadan Fasting on Anaerobic Performance and Recovery Following Short time High Intensity Exercise. J Sports Sci Med. 2007;6:490-7.

2. Moro T, Tinsley G, Bianco A, Marcolin G, Pacelli QF, Battaglia G, Palma A, Gentil P, Neri M, Paoli A. Effects of eight weeks of time-restricted feeding (16/8) on basal metabolism, maximal strength, body composition, inflammation, and cardiovascular risk factors in resistance-trained males. J Trans Med. 2016;14:290.

3. Tinsley GM, Gann JG, La Bounty PM. Intermittent fasting programs and their effects on body composition: implications for weight-restricted sports. Strength Cond J. 2015;37:60-71

4. Bouhlel E, Zaouali M, Miled A, Tabka Z, Bigard X, Shephard R. Ramadan fasting and the GH/IGF-1 axis of trained men during submaximal exercise. Ann Nutr Metab. 2008;52:261-6.

5. Anton SD, Moehl K, Donahoo WT, Marosi K, Lee SA, Mainous AG, 3rd, Leeuwenburgh C, Mattson MP. Flipping the Metabolic Switch: Understanding and Applying the Health Benefits of Fasting. Obesity. 2017.

6. Maughan RJ, Fallah J, Coyle EF. The effects of fasting on metabolism and performance. British J Sports Med. 2010;44:490-4.

7. Varady K. Intermittent versus daily calorie restriction: which diet regimen is more effective for weight loss? Obes Rev. 2011;12:e593-e601.

8. Chow LS, Manoogian ENC, Alvear A, Fleischer JG, Thor H, Dietsche K, Wang Q, Hodges JS, Esch N, Malaeb S, Harindhanavudhi T, Nair KS, Panda S, Mashek DG. TimeRestricted Eating Effects on Body Composition and Metabolic Measures in Humans who are Overweight: A Feasibility Study. Obesity (Silver Spring). 2020;28:860-9.

9. Tinsley GM, Forsse JS, Butler NK, Paoli A, Bane AA, La Bounty PM, Morgan GB, Grandjean PW. Time-restricted feeding in young men performing resistance training: A randomized controlled trial. Euro J Sport Sci. 2017;17:200-7.

10. Anton SD, Moehl K, Donahoo WT, Marosi K, Lee SA, Mainous AG, 3rd, Leeuwenburgh C, Mattson MP. Flipping the Metabolic Switch: Understanding and Applying the Health Benefits of Fasting. Obesity (Silver Spring). 2018;26:254-68. 11. Catenacci VA, Pan Z, Ostendorf D, Brannon S, Gozansky WS, Mattson MP, Martin B, MacLean PS, Melanson EL, Troy Donahoo W. A randomized pilot study comparing zero-calorie alternate-day fasting to daily caloric restriction in adults with obesity. Obesity (Silver Spring). 2016;24:1874-83.

12. Kalam F, Gabel K, Cienfuegos S, Wiseman E, Ezpeleta M, Steward M, Pavlou V, Varady KA. Alternate day fasting combined with a low-carbohydrate diet for weight loss, weight maintenance, and metabolic disease risk reduction. Obes Sci Pract. 2019;5:531-9.

13. Davis CS, Clarke RE, Coulter SN, Rounsefell KN, Walker RE, Rauch CE, Huggins $\mathrm{CE}$, Ryan L. Intermittent energy restriction and weight loss: a systematic review. Eur J Clin Nutr. 2016;70:292-9.

14. Headland ML, Clifton PM, Keogh JB. Effect of intermittent compared to continuous energy restriction on weight loss and weight maintenance after 12 months in healthy overweight or obese adults. Int J Obes. 2019;43:2028-36.

15. Headland ML, Clifton PM, Keogh JB. Impact of intermittent vs. continuous energy restriction on weight and cardiometabolic factors: a 12-month follow-up. Int J Obes (Lond). 2020:1-7.

16. Keogh JB, Pedersen E, Petersen KS, Clifton PM. Effects of intermittent compared to continuous energy restriction on short-term weight loss and long-term weight loss maintenance. Clin Obes. 2014;4:150-6.

17. Correia JM, Santos I, Pezarat-Correia P, Silva AM, Mendonca GV. Effects of Ramadan and Non-ramadan Intermittent Fasting on Body Composition: A Systematic Review and Meta-Analysis. Front Nutr. 2020;7:625240.

18. Heilbronn LK, Smith SR, Martin CK, Anton SD, Ravussin E. Alternate-day fasting in nonobese subjects: effects on body weight, body composition, and energy metabolism. Am J Clin Nutr. 2005;81:69-73.

19. Bhutani S, Klempel MC, Kroeger CM, Trepanowski JF, Varady KA. Alternate day fasting and endurance exercise combine to reduce body weight and favorably alter plasma lipids in obese humans. Obesity (Silver Spring). 2013;21:1370-9.
20. Klempel MC, Kroeger CM, Varady KA. Alternate day fasting (ADF) with a high-fat diet produces similar weight loss and cardio-protection as ADF with a low-fat diet. Metab. 2013;62:137-43.

21. Cho AR, Moon JY, Kim S, An KY, Oh M, Jeon JY, Jung DH, Choi MH, Lee JW. Effects of alternate day fasting and exercise on cholesterol metabolism in overweight or obese adults: A pilot randomized controlled trial. Metab. 2019;93:52-60.

22. Varady KA, Bhutani S, Klempel MC, Kroeger CM, Trepanowski JF, Haus JM, Hoddy KK, Calvo Y. Alternate day fasting for weight loss in normal weight and overweight subjects: a randomized controlled trial. Nutr J. 2013;12:146.

23. Correia JM, Santos I, Pezarat-Correia P, Minderico C, Mendonca GV. Effects of Intermittent Fasting on Specific Exercise Performance Outcomes: A Systematic Review Including Meta-Analysis. Nutrients. 2020;12.

24. Schoenfeld B. Does cardio after an overnight fast maximize fat loss? Strength Cond J. 2011;33:23-5.

25. Tinsley GM, Moore ML, Graybeal AJ, Paoli A, Kim Y, Gonzales JU, Harry JR, VanDusseldorp TA, Kennedy DN, Cruz MR. Time-restricted feeding plus resistance training in active females: a randomized trial. Am J Clin Nutr. 2019;110:628-40.

26. Jabekk PT, Moe IA, Meen HD, Tomten SE, Høstmark AT. Resistance training in overweight women on a ketogenic diet conserved lean body mass while reducing body fat. Nutr Metab. 2010;7:17.

27. Chaouachi A, Coutts AJ, Chamari K, Wong del P, Chaouachi M, Chtara M, Roky R, Amri M. Effect of Ramadan intermittent fasting on aerobic and anaerobic performance and perception of fatigue in male elite judo athletes. J Strength Cond Res. 2009;23:2702-9. 28. Anis C, Leiper JB, Nizar S, Coutts AJ, Karim C. Effects of Ramadan intermittent fasting on sports performance and training: a review. Int $\mathrm{J}$ Sports Physiol Perform. 2009;4:419-34.

29. Roy AS, Bandyopadhyay A. Effect of Ramadan intermittent fasting on selective fitness profile parameters in young untrained Muslim men. BMJ Sport Exerc Med. 2015;1:e000020. 30. Aziz AR, Wahid MF, Png W, Jesuvadian CV. Effects of Ramadan fasting on 60 min of endurance running performance in moderately trained men. Br J Sports Med. 2010;44:516-21.

31. Gueldich H, Zghal F, Borji R, Chtourou H, Sahli S, Rebai H. The effects of Ramadan intermittent fasting on the underlying mechanisms of force production capacity during maximal isometric voluntary contraction. Chronobiol Int. 2019;36:698-708.

32. Ferguson LM, Rossi KA, Ward E, Jadwin E, Miller TA, Miller WC. Effects of caloric restriction and overnight fasting on cycling endurance performance. J Strength Cond Res. 2009;23:560-70.

33. Ramadan J. Does fasting during Ramadan alter body composition, blood constituents and physical performance? Med Princ Pract. 2002;11:41-6.

34. Chtourou H, Hammouda O, Chaouachi A, Chamari K, Souissi N. The effect of time-of-day and Ramadan fasting on anaerobic performances. Int J Sports Med. 2012;33:142-7.

35. Shephard RJ. The Impact of Ramadan Observance upon Athletic Performance. Nutrients. 2012;4:491-505.

36. McMurray RG, Ben-Ezra V, Forsythe WA, Smith AT. Responses of endurancetrained subjects to caloric deficits induced by diet or exercise. Med Sci Sports Exerc. 1985;17:574-9.

37. Sweileh N, Schnitzler A, Hunter GR, Davis B. Body composition and energy metabolism in resting and exercising muslims during Ramadan fast. J Sports Med Phys Fitness. 1992;32:156-63.

38. Ramadan JM, Barac-Nieto M. Cardio-respiratory responses to moderately heavy aerobic exercise during the Ramadan fasts. Saudi Med J. 2000;21:238-44.

39. Brisswalter J, Bouhlel E, Falola JM, Abbiss CR, Vallier JM, Hausswirth C. Effects of Ramadan intermittent fasting on middle-distance running performance in well-trained runners. Clin J Sport Med. 2011;21:422-7.

40. Gueye L, Samb A, Seck D, Cissé F, Camara K, Martineaud J. Influence of a 12 hours-fast on maximal exercise. Scrip Med (Brno). 2004;77:5-8.

41. Racinais S, Periard JD, Li CK, Grantham J. Activity patterns, body composition and muscle function during Ramadan in a Middle-East Muslim country. Int J Sports Med. 2012;33:641-6. 
42. Templeman I, Thompson D, Gonzalez J, Walhin JP, Reeves S, Rogers PJ, Brunstrom JM, Karagounis LG, Tsintzas K, Betts JA. Intermittent fasting, energy balance and associated health outcomes in adults: study protocol for a randomised controlled trial. Trials. 2018;19:86.

43. Ziaee V, Razaei M, Ahmadinejad Z, Shaikh H, Yousefi R, Yarmohammadi L, Bozorgi F, Behjati MJ. The changes of metabolic profile and weight during Ramadan fasting Sing Med J. 2006;47:409-14.

44. Syam AF, Sobur CS, Abdullah M, Makmun D. Ramadan fasting decreases body fat but not protein mass. Int J Endocr Metab. 2016;14.

45. Hajek P, Myers K, Dhanji AR, West O, McRobbie H. Weight change during and after Ramadan fasting. J Public Health. 2012;34:377-81.

46. Bilto YY. Effects of Ramadan fasting on body weight and the biochemical and haematological parameters of the blood. Arab Gulf J Sci Res. 1998;16:1-13.

47. Al-Numair K. Body Weight and Some Biochemical Changes Associated with Ramadan Fasting in Healthy Saudi Men. J Med Sci. 2006;6:112-6.

48. Al-Hourani HM, Atoum MF. Body composition, nutrient intake and physical activity patterns in young women during Ramadan. Sing Med J. 2007;48:906-10.

49. Salehi M, Neghab M. Effects of fasting and a medium calorie balanced diet during the holy month Ramadan on weight, BMI and some blood parameters of overweight males. Pak J Biol Sci. 2007;10:968-71

50. Stannard SR, Thompson MW. The effect of participation in Ramadan on substrate selection during submaximal cycling exercise. J Sci Med Sport. 2008;11:510-7. 51. Mirzaei B, Rahmani-Nia F, Moghadam MG, Ziyaolhagh SJ, Rezaei A. The effect of ramadan fasting on biochemical and performance parameters in collegiate wrestlers. Iran J Basic Med Sci. 2012;15:1215-20.

52. Aloui A, Chaouachi A, Chtourou H, Wong DP, Haddad M, Chamari K, Souissi N. Effects of Ramadan on the diurnal variations of repeated-sprint performance. Int $\mathrm{J}$ Sports Physiol Perform. 2013;8:254-63.

53. Norouzy A, Salehi M, Philippou E, Arabi H, Shiva F, Mehrnoosh S, Mohajeri SM, Mohajer SA, Motaghedi Larijani A, Nematy M. Effect of fasting in Ramadan on body composition and nutritional intake: a prospective study. J Hum Nutr Diet. 2013;26 Suppl 1:97-104. 54. Rohin MA, Rozano N, Abd Hadi N, Mat Nor MN, Abdullah S, Dandinasivara Venkateshaiah M. Anthropometry and body composition status during Ramadan among higher institution learning centre staffs with different body weight status. Sci World J. 2013;2013:308041.

55. el Ati J, Beji C, Danguir J. Increased fat oxidation during Ramadan fasting in healthy women: an adaptative mechanism for body-weight maintenance. Am J Clin Nutr. 1995;62:302-7.

56. Ramadan J, Telahoun G, Al-Zaid NS, Barac-Nieto M. Responses to exercise, fluid, and energy balances during Ramadan in sedentary and active males. Nutrition. 1999;15:735-9.

57. Ramadan J. Does fasting during Ramadan alter body composition, blood constituents and physical performance? Med Princ Pract Int J. 2002;11 Suppl 2:41-6. 58. Haouari M, Haouari-Oukerro F, Sfaxi A, Ben Rayana MC, Kaabachi N, Mbazaa A. How Ramadan fasting affects caloric consumption, body weight, and circadian evolution of cortisol serum levels in young, healthy male volunteers. Horm Metab Res. 2008;40:575-7.

59. Chennaoui M, Desgorces F, Drogou C, Boudjemaa B, Tomaszewski A, Depiesse F, Burnat P, Chalabi H, Gomez-Merino D. Effects of Ramadan fasting on physical performance and metabolic, hormonal, and inflammatory parameters in middle-distance runners. Appl Physiol Nutr Metab 2009;34:587-94.

60. SÜLÜ B, ÖZtÜRk B, GÜVen A, Kiliç K. The Effect of Long-Term Controlled Fasting (The Ramadan Model) on Body Mass Index, Blood Biochemistry and Oxidative Stress Factors. J Med Sci. 2010;30:855-63

61. Trabelsi K, Stannard SR, Ghlissi Z, Maughan RJ, Kallel C, Jamoussi K, Zeghal KM, Hakim A. Effect of fed- versus fasted state resistance training during Ramadan on body composition and selected metabolic parameters in bodybuilders. J Int Soc Sports Nutr. 2013;10:23.

62. Harvie M, Wright C, Pegington M, McMullan D, Mitchell E, Martin B, Cutler RG, Evans G, Whiteside S, Maudsley S, Camandola S, Wang R, Carlson OD, Egan JM,
Mattson MP, Howell A. The effect of intermittent energy and carbohydrate restriction v. daily energy restriction on weight loss and metabolic disease risk markers in overweight women. British J Nutr. 2013;110:1534-47.

63. Mattson MP, Longo VD, Harvie M. Impact of intermittent fasting on health and disease processes. Ageing Res Rev. 2017;39:46-58.

64. Harvie M, Howell A. Potential benefits and harms of intermittent energy restriction and intermittent fasting amongst obese, overweight and normal weight subjects-a narrative review of human and animal evidence. Behav Sci. 2017;7:4

65. Stote KS, Baer DJ, Spears K, Paul DR, Harris GK, Rumpler WV, Strycula P, Najjar SS, Ferrucci L, Ingram DK. A controlled trial of reduced meal frequency without caloric restriction in healthy, normal-weight, middle-aged adults. Am J Clin Nutr. 2007;85:981-8. 66. Hayward S, Outlaw J, Urbina S, Burks B, Holt J, Stone M, Regeski A, Saur M, Ander J, Taylor $\mathrm{L}$. Effects of intermittent fasting on markers of body composition and mood state. J Int Soc Sports Nutr. 2014;11:1-2.

67. De Bock K, Derave W, Eijnde BO, Hesselink MK, Koninckx E, Rose AJ, Schrauwen P, Bonen A, Richter EA, Hespel P. Effect of training in the fasted state on metabolic responses during exercise with carbohydrate intake. J Appl Physiol (1985). 2008;104:1045-55.

68. Zouhal H, Saeidi A, Salhi A, Li H, Essop MF, Laher I, Rhibi F, Amani-Shalamzari S, Ben Abderrahman A. Exercise Training and Fasting: Current Insights. Open Access J Sports Med. 2020;11:1-28.

69. Rittig N, Bach E, Thomsen HH, Møller AB, Hansen J, Johannsen M, Jensen E, Serena A, Jørgensen JO, Richelsen B. Anabolic effects of leucine-rich whey protein, carbohydrate, and soy protein with and without $\beta$-hydroxy- $\beta$-methylbutyrate (HMB) during fasting-induced catabolism: A human randomized crossover trial. Clin Nutr. 2017;36:697-705.

70. Lessan N, Saadane I, Alkaf B, Hambly C, Buckley AJ, Finer N, Speakman JR, Barakat MT. The effects of Ramadan fasting on activity and energy expenditure. Am J Clin Nutr. 2018;107:54-61.

71. Alsubheen SA, Ismail M, Baker A, Blair J, Adebayo A, Kelly L, Chandurkar V, Cheema S, Joanisse DR, Basset FA. The effects of diurnal Ramadan fasting on energy expenditure and substrate oxidation in healthy men. British J Nutr. 2017;118:1023-30. 72. Fernando HA, Zibellini J, Harris RA, Seimon RV, Sainsbury A. Effect of Ramadan Fasting on Weight and Body Composition in Healthy Non-Athlete Adults: A Systematic Review and Meta-Analysis. Nutrients. 2019;11:478.

73. Kreitzman SN, Coxon AY, Szaz KF. Glycogen storage: illusions of easy weight loss, excessive weight regain, and distortions in estimates of body composition. Am J Clin Nutr. 1992;56:292S-3S.

74. Cava E, Yeat NC, Mittendorfer B. Preserving Healthy Muscle during Weight Loss. Adv Nutr. 2017;8:511-9.

75. Stannard SR, Buckley AJ, Edge JA, Thompson MW. Adaptations to skeletal muscle with endurance exercise training in the acutely fed versus overnight-fasted state. J Sci Med Sport. 2010;13:465-9.

76. Van Proeyen K, Szlufcik K, Nielens H, Ramaekers M, Hespel P. Beneficial metabolic adaptations due to endurance exercise training in the fasted state. J Appl Physiol. 2011;110:236-45.

77. Charlot K, Pichon A, Chapelot D. Effets de l'entraînement à jeun sur la VO2 max, l'oxydation des lipides et la performance aérobie chez des jeunes hommes modérément entraînés. Sci Sports. 2016;31:166-71.

78. Terada T, Toghi Eshghi SR, Liubaoerjijin Y, Kennedy M, Myette-Cote E, Fletcher K, Boule NG. Overnight fasting compromises exercise intensity and volume during sprint interval training but improves high-intensity aerobic endurance. J Sports Med Phys Fitness. 2019;59:357-65.

79. Stocks B, Dent JR, Ogden HB, Zemp M, Philp A. Postexercise skeletal muscle signaling responses to moderate- to high-intensity steady-state exercise in the fed or fasted state. Am J Physiol Endocr Metab. 2019;316:E230-E8.

80. Moro T, Tinsley G, Longo G, Grigoletto D, Bianco A, Ferraris C, Guglielmetti M, Veneto A, Tagliabue A, Marcolin G, Paoli A. Time-restricted eating effects on performance, immune function, and body composition in elite cyclists: a randomized controlled trial. J Int Soc Sports Nutr. 2020;17:65. 
81. Cherif A, Meeusen R, Farooq A, Ryu J, Fenneni M, Nikolovski Z, Elshafie S, Chamari K, Roelands B. Three Days of Intermittent Fasting: Repeated-Sprint Performance Decreased by Vertical-Stiffness Impairment. Int J Sports Physiol Perform. 2017;12:287-94.

82. Naharudin MNB, Yusof A. The effect of 10 days of intermittent fasting on Wingate anaerobic power and prolonged high-intensity time-to-exhaustion cycling performance. Europ J Sport Sci. 2018;18:667-76.

83. Kirkendall DT, Leiper JB, Bartagi Z, Dvorak J, Zerguini Y. The influence of Ramadan on physical performance measures in young Muslim footballers. J Sports Sci. 2008;26 Suppl 3:S15-27.

84. Aziz AR, Che Muhamad AM, Roslan SR, Ghulam Mohamed N, Singh R, Chia MYH. Poorer Intermittent Sprints Performance in Ramadan-Fasted Muslim Footballers despite Controlling for Pre-Exercise Dietary Intake, Sleep and Training Load. Sports (Basel). 2017;5:4.

85. Aziz AR, Che Muhamad AM, Roslan SR, Ghulam Mohamed N, Singh R, Chia MYH. Poorer Intermittent Sprints Performance in Ramadan-Fasted Muslim Footballers despite Controlling for Pre-Exercise Dietary Intake, Sleep and Training Load. Sports (Basel). 2017;5.

86. Dannecker EA, Liu Y, Rector RS, Thomas TR, Sayers SP, Leeuwenburgh C, Ray BK. The effect of fasting on indicators of muscle damage. Exp Gerontol. 2013;48:1101-6. 87. Stratton MT, Tinsley GM, Alesi MG, Hester GM, Olmos AA, Serafini PR, Modjeski AS, Mangine GT, King K, Savage SN. Four Weeks of Time-Restricted Feeding Combined with Resistance Training Does Not Differentially Influence Measures of Body Composition, Muscle Performance, Resting Energy Expenditure, and Blood Biomarkers. Nutrients. 2020;12:1126.

88. Bouhlel H, Latiri I, Zarrrouk N, Bigard X, Shephard R, Tabka Z, Bouhlel E. Effet du jeûne du Ramadan et de l'exercice maximal sur le temps de réaction simple et de choix chez des sujets entraînés. Sci Sports. 2014;29:131-7.

89. Maughan RJ, Bartagi Z, Dvorak J, Zerguini Y. Dietary intake and body composition of football players during the holy month of Ramadan. J Sports Sci. 2008;26 Suppl 3:S29-38.

90. Bouhlel E, Denguezli M, Zaouali M, Tabka Z, Shephard RJ. Ramadan fasting's effect on plasma leptin, adiponectin concentrations, and body composition in trained young men. Int J Sport Nutr Exerc Metab. 2008;18:617-27.

91. Kerndt PR, Naughton JL, Driscoll CE, Loxterkamp DA. Fasting: the history, pathophysiology and complications. West J Med. 1982;137:379-99.

92. Carlson MG, Snead WL, Campbell PJ. Fuel and energy metabolism in fasting humans. Am J Clin Nutr. 1994;60:29-36.

93. Mohsenzadeh A, Baghbani MM, Ranjbar R. The effect of endurance training accompanied by Ramadan fasting on lipid profiles and body composition in men. $J$ Health Res 2013.

94. Souissi N, Souissi H, Sahli S, Tabka Z, Dogui M, Ati J, Davenne D. Effect of Ramadan on the diurnal variation in short-term high power output. Chronobiol Int. 2007;24:991-1007.

95. Aloui A, Driss T, Baklouti H, Jaafar H, Hammouda O, Chamari K, Souissi N. Repeated-sprint training in the fasted state during Ramadan: morning or evening training? J Sports Med Phys Fitness. 2018;58:990-7.

96. Bouhlel H, Shepard R, Gmada N, Aouichaoui C, Peres G, Tabka Z, Bouhlel E. Effect of Ramadan observance on maximal muscular performance of trained men. Clin J Sport Med. 2013;23:222-7.

97. Teo W, Newton MJ, McGuigan MR. Circadian rhythms in exercise performance: implications for hormonal and muscular adaptation. J Sports Sci Med. 2011;10:600-6. 98. Aziz AR, Slater GJ, Chia MYH, Teh KC. Effects of Ramadan fasting on training induced adaptations to a seven-week high-intensity interval exercise programme. Sci Sports. 2012;27:31-8.

99. Meckel Y, Ismaeel A, Eliakim A. The effect of the Ramadan fast on physical performance and dietary habits in adolescent soccer players. Eur J Appl Physiol. 2008;102:651-7.

100. Leiper JB, Molla AM, Molla AM. Effects on health of fluid restriction during fasting

in Ramadan. Eur J Clin Nutr. 2003;57 Suppl 2:S30-8.
101. Zarrouk N, Hammouda O, Latiri I, Adala H, Bouhlel E, Rebai H, Dogui M. Ramadan fasting does not adversely affect neuromuscular performances and reaction times in trained karate athletes. J Int Soc Sports Nutr. 2016;13:18. 